\title{
Classical conditioning in patients with severe memory problems
}

\author{
IRENE DAUM,* SHELLEY CHANNON, * ANTHONY G M CANAVAN†
}

From the Department of Psychology, Institute of Psychiatry, London, UK* and Institute of Psychology, University of Tübingen, Tübingen, FRG†

SUMMARY Classical conditioning is one of the most fundamental forms of learning, and yet little is known regarding the effects of brain injury on conditioning processes in humans. Three patients with temporal lobe lesions and severe memory problems were therefore assessed in terms of eyeblink conditioning, extinction, discrimination and reversal learning, and in one patient electrodermal conditioning was also investigated. The acquisition of conditioned responses was seen to be intact, but the evidence regarding extinction was ambiguous. All of the patients were impaired in discrimination learning and also reversal learning.

Classical conditioning is one of the most fundamental forms of learning, and has been important in theories of both experimental psychology ${ }^{1}$ and clinical psychology. ${ }^{2}$ Classical conditioning techniques have also been extensively applied in pharmacological research ${ }^{3}$ and have formed the basis of many early neuropsychological investigations into the effects of brain lesions in animals. ${ }^{4} \mathrm{It}$ is known, for instance, that classical conditioning can survive even complete cerebral decortication in the rat and the rabbit, ${ }^{56}$ although in these same animals classical conditioning may be abolished or impaired following lesions of the cerebellum $^{7}$ or limbic structures. ${ }^{89}$ It is therefore surprising that little is known about classical conditioning in the brain-injured human, especially since a great deal is known about classical conditioning in healthy subjects. ${ }^{1011}$

To our knowledge only two attempts have been made to study classical conditioning in patients with brain lesions. The first was a single case study involving H.M., the well-documented patient who suffered total anterograde amnesia following bilateral mesial resection of the temporal lobes. ${ }^{12}$ H.M., however, failed to show phasic electrodermal responses to the unconditioned stimulus (UCS), an electroshock, in a procedure designed to condition the galvanic skin response to a neutral stimulus, and thus

Address for reprint requests: Irene Daum, Department of Psychology, Institute of Psychiatry, De Crespigny Park, Denmark Hill, London SE5 8AF, UK.

Received 10 May 1988 and in revised form 4 August 1988. Accepted 8 August 1988 the experiment had to be terminated. ${ }^{13}$ In the only other study in this field, Weiskrantz and Warrington ${ }^{14}$ demonstrated unimpaired classical conditioning in two severely amnesic patients, using an eyeblink conditioning paradigm. The patients showed adequate development of conditioned responses (CRs) despite being unable to report from memory the actual stimulus contingencies.

This second study raised further issues. First, the subjects' eyeblink movements were not directly recorded, but were evaluated on the basis of videos. This did not allow a detailed analysis of latencies of blink onsets which is crucial in determining the nature of the blinks. ${ }^{10}$ Second, the criterion for the occurrence of a CR was blink onsets starting from $100 \mathrm{~ms}$ after the onset of a conditioned stimulus (CS) which consisted of a combined light-tone signal. Recent studies, however, use much stricter latency criteria for the occurrence of an eyeblink CR. ${ }^{15}$ Blinks with onsets up to $250 \mathrm{~ms}$ after CS onset might have to be regarded as reflex blinks to the conditioned stimulus rather than conditioned responses. ${ }^{10}$

The present study therefore aimed to replicate the findings of Weiskrantz and Warrington, using more refined recording and analysis techniques, and employing electrodermal conditioning as well as eyeblink conditioning. In addition, conditioning performance was extended to the investigation of extinction, discrimination and reversal learning paradigms, since these have been found to be more sensitive to impairments in animals with lesions to the limbic system. ${ }^{89}$ The patients investigated displayed temporal lobe damage and severe memory problems. 


\section{Methods}

\section{Subjects}

Patient 1 was a 63 year old hospitalised housewife with a history of epilepsy since the age of 38 and a history of alcohol abuse. The EEG showed diffuse slowing over both hemispheres with left-sided discharges over temporal and parietal regions and independent discharges from the anterior part of the right temporal lobe. Her verbal IQ (VIQ) (calculated according to Canavan, Dunn and McMillan ${ }^{16}$ ) on the WAISR was 88, whilst her Performance IQ (PIQ) (calculated according to Canavan et $\mathrm{al}^{16}$ ) was 73. Performance on the Wechsler Memory Passages ${ }^{17}$ was moderately impaired for immediate recall (score 6.5 on Passage $A$ and 4.0 on passage B). The patient could not recall any item after 1 hour (expected: 9 items across the two passages ${ }^{17}$ ). Whilst copying of the Rey Figure ${ }^{17}$ was adequate, delayed recall was severely impaired $\left(13 \%\right.$, expected: $\left.47 \%{ }^{17}\right)$ and consisted of a vague outline which might have reflected the representation of virtually any spatial material. Medication consisted of phenytoin $300 \mathrm{mg}$.

Patient 2 was a 22 year old unemployed female who had suffered from encephalitis at the age of 12 . Subsequently she developed epileptic seizures and severe memory deficits. The EEG showed focal spikes in the left and occasionally in the right temporal region. Both VIQ and PIQ were 70. The patient showed severe bilateral memory dysfunction superimposed on the borderline IQ. She remembered 1 item each on Passages A and B from the Wechsler Memory Scale on immediate recall, and 1 item on delayed recall (expected: 4 items $\left.{ }^{17}\right)$. She scored 4 correct (expected 6) and 13 errors (expected 6) on the Benton test. ${ }^{18}$ Copying skills on the Rey Figure were intact (43/47), whilst delayed recall was severely impaired (12\%, expected: $\left.59 \%{ }^{17}\right)$. At the time of testing she was medicated with carbamazepine $\mathbf{4 0 0} \mathrm{mg}$ and valproate $1500 \mathrm{mg}$.

Patient 3 was a 30 year old unemployed male who suffered from epileptic seizures following febrile convulsions in infancy. Whilst the EEG was unremarkable, CT showed anterior hypoplasia of the right temporal lobe, and a similar milder abnormality in the left temporal lobe. VIQ was 112 , PIQ was 70. Immediate recall of the Wechsler Passages was moderately impaired (A: 7.0, B: 6.5) whereas delayed recall was severely impaired ( 2 items in total, expected: 15 items ${ }^{17}$ ). Immediate visuospatial recall (Benton Test) comprised 5 correct responses (expected 6) and 8 errors (expected 6). Delayed visuospatial recall was severely impaired $(23.4 \%$ of the Rey Figure, expected: $56 \%{ }^{17}$ ). This patient was receiving phenytoin $400 \mathrm{mg}$ and valproate $1600 \mathrm{mg}$.

\section{Experimental schedule}

Patients were presented with a series of reinforced and unreinforced trials, using a reinforcement schedule of 2:1. A reinforced trial consisted of a $6 \mathrm{~s}$ signal light $(\mathrm{S}+)$ followed by a $1 \mathrm{~s}$ gap and an $800 \mathrm{~ms}$ tone $(1000 \mathrm{~Hz}, 65 \mathrm{db}$ sound pressure level) presented binaurally through headphones. The unconditioned stimulus (UCS) was a corneal airpuff $(41 \mathrm{kPa})$ to the right eye, presented through a nozzle mounted on the goggles worn by the subject. The puff onset was $720 \mathrm{~ms}$ after the tone (conditioned stimulus, CS), lasting $80 \mathrm{~ms}$ and terminating with the tone. Mean inter-trial interval was $20 \mathrm{~s}$. An unreinforced trial consisted of a different colour light (S-), a $1 \mathrm{~s} \mathrm{gap}$ and an identical tone (no airpuff). Red and green lights served as the signal lights, mounted on a white panel about $1.5 \mathrm{~m}$ in front of the subject.

\section{Apparatus and recording}

Subjects were seated in a darkened room in a comfortable armchair. Throughout the testing session, the subjects' eyelid movements were monitored continuously by means of a cadmium selenide photocell mounted on the right eyepiece of a pair of goggles worn by the subject and recorded on magnetic tape for off-line computer analysis. Scoring of the waveform of the eyelid data was carried out by computer using a method consisting of detecting changes in the slope of the curve as a function of time. ${ }^{19}$ A conditioned response was defined as any departure of $0.4 \mathrm{~cm}$ or more from a stable baseline occurring within the latency range of $390-720 \mathrm{~ms}$ after tone onset. This CR latency band had been established in a large scale study using normal volunteers ${ }^{20}$ and differs from the criterion used by Warrington and Weiskrantz, which was any incomplete closure of the eyelid occurring 100 ms or later after CS onset (a tone-light combination). The present criterion was chosen to avoid the scoring of reflex blinks to the conditioned stimulus, which might occur up to $250 \mathrm{~ms}$ after stimulus onset. ${ }^{10}$

For Patient 1, skin resistance was recorded from the middle phalanges of the index finger and the middle finger of the left hand using $\mathrm{Ag} / \mathrm{AgCl}$ electrodes and an electrode paste which contained $0.05 \mathrm{M} \mathrm{NaCl}$ in a cellulose base. The current was $10 \mu \mathrm{A}$. Skin resistance was stored on tape and submitted to off-line analysis using the software package SARA. ${ }^{19}$

\section{Procedure}

Patients were told that they were taking part in a memor test. They would see some lights, hear some noises anc occasionally get a puff of air to the eye which would not be harmful or painful. They were instructed not to close thei eyes deliberately but to watch all the things that happened because they would be questioned about the procedure afterwards.

Patient 1 received a simple conditioning schedule consisting of 30 trials (20 reinforced trials $(S+)$ and 10 unreinforced trials $(\mathbf{S}-)$ ), in sequences of $\mathbf{S}+\mathbf{S}+\mathbf{S}-$. This acquisition phase was followed by an interview consisting of initial free recall ("Tell me what happened in the test") and a sequence of structured questions to assess memory and awareness about stimulus contingencies (such as "Did you see any lights?", "Did the bleeps come before or after the lights?" "Was the bleep always followed by an airpuff?", "When the red lights came on, was there an airpuff after the bleep?"). This interview lasted about $10 \mathrm{~min}$ and was followed by six reacquisition trials and 12 extinction trials. Then a 30 trials reversal phase followed in which the light that previously signalled the "tone only" condition now signalled the tonepuff combination. The light that previously signalled tone/ puff now signalled the "tone only" trials. Following reversal, a final extinction phase was presented. Each extinction stage and the reversal stage were followed by interviews about stimulus contingencies.

Patients 2 and 3 received 36 conditioning trials ( 24 reinforced $(\mathrm{S}+), 12$ unreinforced $(\mathrm{S}-)$ trials), with $\mathrm{S}+$ and $\mathrm{S}-$ trials presented in random order. Acquisition was followed by an interview (see above) and 36 reversal trials. The reversal 
learning stage was again followed by an interview about stimulus contingencies and an extinction stage consisting of six reacquisition and 12 extinction trials. Testing ended with a final interview about the extinction stage. Patient 3 had taken part in a pilot investigation for the present study involving eyelid conditioning which had been carried out 3 months before the present testing. On second testing he said that he remembered having been in the laboratory before "for some tests", but he could not remember any details about the tests.

\section{Results}

First occurrence of a conditioned response

The first conditioned response (CR) occurred on trial 4 in the initial acquisition phase and on trial 3 in the reversal learning phase for Patient 1 . Patient 2 showed the first CR on trial 5 in both the acquisition phase and the reversal phase. In the case of Patient 3, the first CR occurred on trial 2 in the acquisition phase and on the first trial in the reversal stage. In comparison, Kayata ${ }^{20}$ reported first CRs to occur on average on trials 5 to 10 using a similar paradigm in a study with normal volunteers.

Development of conditioned responses over blocks of trials

The number of conditioned responses (CRs) to the tone (combining reinforced and unreinforced trials) over blocks of six trials is presented in the fig. In the initial acquisition phase, all patients showed a typical learning curve, with response rates increasing from earlier to later blocks of trials. Patient 3 started off with an exceptionally high response rate in the first block which might be partly accounted for by his previous experience with a similar task. In the reversal stage, Patient 3 showed a consistently high response rate from the beginning which indicates transfer of learning across the 10 min interview stage. During reversal, Patient 2 displayed a curve which was similar to the initial acquisition curve. The response rate of Patient 1 during reversal was very low and showed some decrease over blocks which might be partly due to an inhibitory effect from the preceding extinction phase. The CR frequencies of Patients 2 and 3 were very high, considering that only $67 \%$ of tones were reinforced by an airpuff.

\section{Extinction}

Patient 1, who received an extinction stage directly after acquisition and also after reversal, showed a decrease in CR rates from acquisition to the first extinction stage, but no further decrease from reacquisition (block 1 ) to the proper extinction trials (blocks 2 and 3) (see fig). In the second extinction stage, an initial decrease in CR rates was followed by a subsequent increase. Patients 2 and 3 received only one
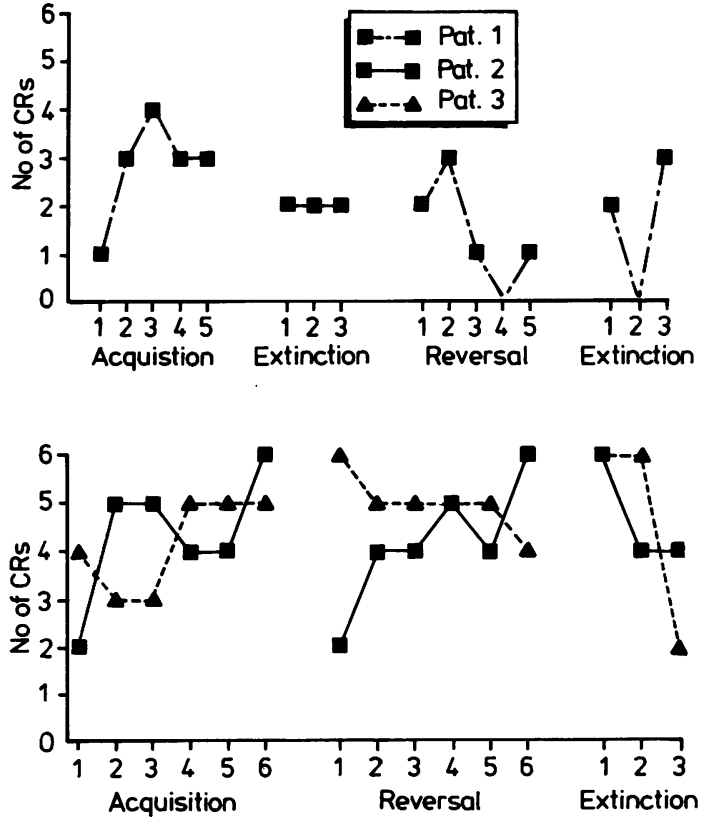

Blocks of 6 trials

Fig Number of conditioned responses to the tone during the different stages of the experiment.

extinction phase, after reversal. Patient 2 showed a small decrease from reacquisition to extinction, though the CR frequency is still very high at the end of extinction. Patient 3 showed a large decrease across reacquisition and the earlier extinction trials to the later trials.

\section{Discrimination}

When responses on $S+$ and $S-$ trials were considered separately, the following pattern emerged: during acquisition, all patients showed similar $C R$ rates on both $\mathrm{S}+$ and $\mathrm{S}-$ trials or even a somewhat higher response rate on $S-$ trials (see table). The response rates for $\mathbf{S}+$ trials are similar to those observed for healthy volunteers ${ }^{20}$, but the $S-$ response rates found in the patients are considerably higher.

\section{Reversal}

The CR rates for the reversal learning stage are presented in the table. As in discrimination, the patients had similar CR rates on both trial conditions, or showed higher responding on $\mathrm{S}-$ trials.

\section{Awareness of stimulus contingencies}

All patients correctly recalled the colours of the signal lights and reported that they had heard tones and received air puffs to the eye. Patients 2 and 3 answered correctly that the lights preceded the tones, and that 
Table Response frequencies on reinforced $(S+)$ and unreinforced (S-) trials for Subjects 1 (P1), 2 (P2) and 3 (P3)

\begin{tabular}{lllll}
\hline & & $P 1$ & $P 2$ & $P 3$ \\
\hline \multirow{2}{*}{ Discrimination } & S - & $45 \%$ & $67 \%$ & $42 \%$ \\
\multirow{2}{*}{ Reversal } & S - & $50 \%$ & $83 \%$ & $67 \%$ \\
& S - & $10 \%$ & $67 \%$ & $75 \%$ \\
& & $50 \%$ & $75 \%$ & $92 \%$ \\
\hline
\end{tabular}

the air puffs came after the tones, whereas Patient 1 was unsure about these sequences. Only Patient 3 could state the rule that red lights were followed by tone/airpuff and green lights by tone alone (and vice versa for reversal). Patients 1 and 2 said in the respective interviews that both coloured lights were probably followed by an airpuff. Patient 2 correctly answered that there were no more airpuffs at the end of the extinction stage, whereas patients 1 and 3 were unsure.

\section{Electrodermal responding}

Patient 1 showed 10 electrodermal orienting responses (ORs) (onset range 1 to $3 \mathrm{~s}$ after light onset) to the lights ( 5 to red lights, 5 to green lights) during the 30 acquisition trials. 7 ORs occurred during reversal learning ( 4 to red lights, 3 to green lights). She also displayed 2 ORs in the first and 4 in the second extinction stage.

The airpuff resulted in unconditioned electrodermal responses to the tone on 4 trials in acquisition and five trials during reversal (onset range 1.8 to $3.4 \mathrm{~s}$ after puff onset).

Conditioned responses (CRs) to the tones were responses with onset 0.5 to $1.7 \mathrm{~s}$ after the tone onset (onset range based on Kayata ${ }^{20}$ ). During initial acquisition, the subject showed five electrodermal CRs on $\mathrm{S}+$ trials and no response on $\mathrm{S}-$ trials. During reversal, 2 CRs occurred on $S+$ trials and 2 on $S-$ trials. In the extinction trials, 2 CRs occurred in initial extinction and 1 in the final extinction stage.

\section{Discussion}

The present findings provide evidence for unimpaired acquisition of classical conditioning in patients with temporal lobe damage and severe memory problems. Patients show similar speed of conditioning and similar learning curves to those normally observed in classical eyelid conditioning experiments. There was evidence of transfer of learning across interview periods of approximately $10 \mathrm{~min}$ (in Patient 2 from reversal to extinction and in Patient 3 across all interview periods). Additionally, the rapid conditioning and the high initial rate of conditioning in a patient who had previous experience with eyelid conditioning about 3 months prior to the present testing might be considered as evidence for the long-term retention of conditioned responding. This has been observed in normal subjects. ${ }^{21}$

The present results thus confirm the observations by Weiskrantz and Warrington, ${ }^{14}$ who reported unimpaired classical conditioning in amnesic patients. The present observations also confirm that unconditioned electrodermal responses can be evoked in patients with severe memory problems. Additionally, electrodermal orienting responses to signal lights occurred and conditioned electrodermal responses developed in a similar way to those reported for normal subjects. ${ }^{20}$

The question as to whether normal extinction occurred, cannot be answered in the light of the present observations. Patient 3 showed clear evidence of extinction, whereas the extinction curves for Patients 1 and 2 are ambiguous and do not demonstrate decreasing responding over trials.

It clearly emerged from the present results that none of the patients was able to show discrimination learning with regard to conditioned responses. The poor performance is mainly due to the very high response rates on $\mathrm{S}$ - trials. Performance is unrelated to awareness of the stimulus contingencies. These findings might be explained by the fact that the patients associated the airpuff with the tone, but dido not make this association conditional on a preceding stimulus (the red or green light). This procedure leads to responding to the tone, irrespective of whether the tone had been preceded by a $S+$ or a $S-$. The results would thus indicate a failure on conditional responding which is in line with the animal literature on hippocampal lesions, ${ }^{9}$ from which we assume the patients suffer, given the temporal lobe damage substantiated by EEGs and CT.

Interestingly, Patient 1 showed some evidence of discrimination as regards electrodermal CRs in the initial acquisition stage, although unaware of the stimulus contingencies. It is difficult to evaluate the significance of electrodermal CRs, as they do not serve a specific purpose. In comparison, conditioned eyeblinks lead to the closure of the eyelid and thus to an avoidance of the direct impact of an aversive stimulus (airpuff), whereas increased electrodermal responding mirrors a rather non-specific increase in arousal. Further experimental evidence is needed before any conclusions on electrodermal discrimination can be drawn. The same holds for reversal learning: as no discrimination learning occurred in the first place, the results from the reversal learning stage of the experiment cannot be discussed in the light of the "reversal learning" concept.

Further work is needed to clarify whether patients with temporal lobe damage would eventually display a conditional discrimination given considerably more learning trials than employed in the present paradigm 
and usually needed for normal subjects. A further issue of interest is how far awareness of stimulus contingencies might influence the discrimination performance within a classical conditioning paradigm in patients with memory deficits.

The authors thank Prof J A Gray for consultation and useful discussion.

\section{References}

1 Gray JA. Elements of a Two-process Theory of Learning. London: Academic Press, 1975.

2 Eysenck HJ. The conditioning model of neurosis. Behav Brain Sci 1979;2:155-99.

3 Iversen SD, Inversen LL. Behavioural Pharmacology. 2nd ed. New York: Oxford University Press, 1981.

4 Mackintosh NJ. The Psychology of Animal Learning. London: Academic Press, 1974.

5 Oakley DA, Russell IS. Subcortical storage of Pavlovian conditioning in the rabbit. Physiol Behav 1977;18:931-7.

6 Oakley DA, Eames LC, Jacobs JL, Davey GLC, Cleland G. Signal-centred action patterns in rats without neocortex in a Pavlovian conditioning situation. Physiol Psychol 1981;9: 135-44.

7 Yeo C, Hardiman M, Glickstein M. Classical conditioning of the nictitating membrane response of the rabbit. II. Lesions of cerebellar cortex. Exp Brain Res 1985;60:99-113.

8 Orr WB, Berger TW. Hippocampectomy disrupts the topography of conditioned nictitating membrane responses during reversal learning. Behav Neurosci 1985;99:35-45.

9 Ross WT, Orr WB, Holland PC, Berger TW. Hippocampectomy disrupts the acquisition and retention of learned conditional responding. Behav Neurosci 1984;98:211-25.

10 Martin I, Levy AB. The Genesis of the Classically Conditioned Response. Oxford: Pergamon Press, 1969.

11 Davey G, ed. Cognitive Processes and Pavlovian Conditioning in Humans. Chichester: John Wiley, 1987.

12 Milner B, Corkin S, Teuber HL. Further analysis of the hippocampal amnesic syndrome: 14-year follow-up study of HM. Neuropsychologia 1968;6:215-34.

13 Kolb IQ, Whishaw WB. Fundamentals of Human Neuropsychology. San Francisco: Freeman, 1985.

14 Weiskrantz L, Warrington EK. Conditioning in amnesic patients. Neuropsychologia 1979;17:187-94.

15 Frcka G, Martin I. Is there - or is there not - an influence of impulsiveness on classical eyelid conditioning? Personality and Individual Differences 1987;8:241-52.

16 Canavan AGM, Dunn G, McMillan T. Principal components analysis of the WAIS-R. Br J Clin Psychol 1986;25:81-5.

17 Powell GE. The relationship between intelligence and verbal and spatial' memory. J Clin Psychol 1979;35:336-40.

18 Benton AL. The Revised Visual Retention Test. New York: The Psychological Corporation, 1955.

19 Law LN, Levey AB, Martin I. Response detection and measurement. In: Martin I, Venables PH, eds. Techniques in Psychophysiology. Chichester: Wiley, 1980:629-63.

20 Kayata L. Awareness and Human Learning. Unpublished doctoral dissertation. University of London, 1987.

21 Hilgard ER, Humphries LG. The retention of conditioned discrimination in man. J Gen Psychol 1938;19:111-25. 